\title{
Glass Ceramic CAD/CAM crowns and severely altered posterior teeth: a three levels study
}

\author{
Michel Fages ${ }^{1} \cdot$ Stephane Corn $^{2} \cdot$ Pierre Slangen $^{3} \cdot$ Jacques Raynal $^{4} \cdot$ Patrick Ienny $^{2} \cdot$ \\ Kinga Turzo $^{5} \cdot$ Frederic Cuisinier $^{1} \cdot$ Jean-Cédric Durand $^{1}$
}

\begin{abstract}
For many practitioners, longevity of full glass ceramic crowns in the posterior area, molars and premolars, remains a real challenge. The purpose of this article is to identify and evaluate the parameters that can significantly influence their resistance when preparing a tooth. The analysis proposed in this article relies on interrelated studies conducted at three levels: in vitro (mechanical tests), in silico (finite elements simulations) and in vivo (clinical survival rates). The in vitro and the in silico studies proved that an appropriate variation of the geometric design of the preparations enables to increase up to $80 \%$ the mechanical strength of ceramic reconstructions. The in vivo clinical study of CAD/CAM full ceramic crowns was performed in accordance with the principles stated within the in vitro and the in silico studies and provided a $98.97 \%$ success rate over a 6 years period. The variations of geometric design parameters for dental preparation allows for reconstructions with a mechanical breaking up to $80 \%$ higher than that of a non-appropriate combination. These results are confirmed in clinical practice.
\end{abstract}

Jean-Cédric Durand

jean-cedric.durand@umontpellier.fr

1 LBN (Laboratoire de Bioingénierie et Nanosciences) EA4203, Univ. Montpellier, Montpellier, France

2 C2MA (Materials Center), Ecole des mines d'Alès, Alès, France

3 LGEI (Laboratory of Industrial Environment Engineering), Ecole des mines d'Alès, Alès, France

4 Department of Prosthodontics, Faculty of Odontology, Univ. Montpellier, Montpellier, France

5 Department of Oral Biology and Experimental Dental Research, Faculty of Dentistry, University of Szeged, Szeged, Hungary
Graphical Abstract

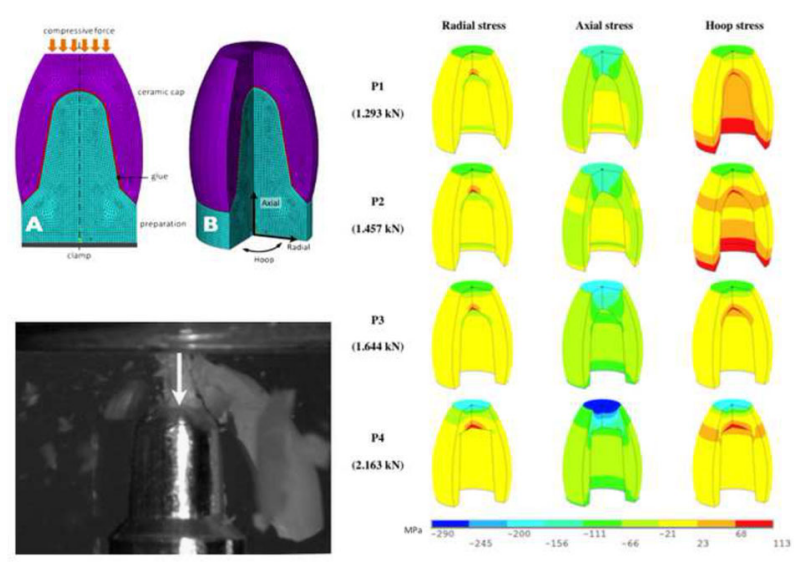

\section{Introduction}

The start of the fatal "Molar life cycle", also defined as the "Cycle of Death" by Simonsen [1, 2], includes a succession of increasingly invasive and destructive treatments, wider restorations and eventual loss of the tooth. Originally, full dental crowns were commonly used and their preparations were based on the mechanistic principles of retention/stabilization [3]. Dental preparations should respond to criteria based on fixing by micro-cottering and the use of mineral cement [3]. At this time, for restorations of lower extended, the mutilating concept of "prophylactic extension" that aims to ensure the complete removal of infected tissue was commonly accepted. Furthermore, implantology could create the dangerous illusion that it was an option to 
prematurely extract teeth as soon as there was sufficient bone volume [4] and replace them with artificial restorations. The cost of implants, medical contraindications, and the appearance of peri-implantitis in recent years have tempered this point of view [5]. With the development of new ceramics and adhesives in the 1990's [6], Magne [7, 8] proposed the use in dentistry of the biomimetic concept developed by Otto Schmitt [9]. Nature proposes models that should be copied; thus, restorations made in accordance to biomimetic features should reproduce the behavior of the tooth under stress. Although this principle seems logical and attractive, $88 \%$ of inventions are unable to fully copy nature [10]. Tooth architecture is sophisticated and complex [11], thus replacing a fragment while avoiding subsequent dysfunctions is a significant challenge.

Observation of teeth and the DEJ (dentin-enamel junction) under mechanical loading shows a complex adaptability to mechanical stress $[12,13]$. Over time, damages induced by variable loadings can alter reconstructions by degradation of either the restoration material itself the adhesive junction between the tooth and the material.

Today, CAD/CAM (Computer-Aided Design/ComputerAided Manufacturing) methods [14] manufacture extremely accurate ceramic reconstructions. To achieve an ideal reconstruction, the practitioner must consider the physical properties of ceramic and their variations in close proximity to enamel, but also mechanical stresses and the geometry of the tooth preparation in order to comply a minimally invasive paradigm.

For severely damaged teeth, the feldspathic ceramic cap appears to be the "perfect restoration" [15], with a wear coefficient and aesthetic close to enamel. The glue joint mimics the DEJ [16]. However, many clinicians do not recommend glued full cap ceramic restorations because of

Table 1 The four preparation designs

\begin{tabular}{|c|c|c|c|c|c|}
\hline Preparations & & $\mathrm{P} 1$ & $\mathrm{P} 2$ & P3 & $\mathrm{P} 4$ \\
\hline \multirow{4}{*}{$\begin{array}{l}\text { Fixed } \\
\text { dimensions }\end{array}$} & $\mathrm{D} 1$ (mm) & 6 & 6 & 6 & 6 \\
\hline & $\mathrm{D} 2(\mathrm{~mm})$ & 4.2 & 4.2 & 4.2 & 4.2 \\
\hline & $\mathrm{H}(\mathrm{mm})$ & 6 & 6 & 6 & 6 \\
\hline & $\mathrm{L}(\mathrm{mm})$ & 0.9 & 0.9 & 0.9 & 0.9 \\
\hline \multirow{3}{*}{$\begin{array}{l}\text { Variables } \\
\text { dimensions }\end{array}$} & TOC $\left(^{\circ}\right)$ & 21 & 7 & 7 & 7 \\
\hline & $\begin{array}{l}\mathrm{COF} \\
\left(\mathrm{mm}^{-1}\right)\end{array}$ & 0.77 & 0.53 & 0.53 & 0 \\
\hline & $\mathrm{FL}\left({ }^{\circ}\right)$ & 45 & 45 & 90 & 90 \\
\hline
\end{tabular}

potential ceramic fractures, especially in the posterior area $[17,18]$. Previously, tooth preparation concepts were not based on both mechanical analyses and a less invasive preparation. The main idea is that optimizing the balance between forces and geometry for ceramic crowns while preserving the underlying substrate should bring on a complete shift in the current restorative dentistry paradigm.

To properly assess the influence of several elements such as the preparation geometry, the characteristics of the reconstruction material and the effects of mechanical stress on glass ceramic, it seemed necessary to conduct a study at three levels: in silico, in vitro and in vivo. In silico numerical simulations determined the most favorable preparation geometry, which was validated by in vitro tests and confirmed by a clinical study.

\section{Materials and methods}

\subsection{In vitro study}

\subsubsection{Preparation models}

Four preparation designs designated as P1, P2, P3, and P4 were produced with Catia V5 software (Dassault Systems, Vélizy-Villacoublay, France). As shown in Table 1, four fixed dimensions were used for all of the preparations: D1 $=$ total diameter, $\mathrm{D} 2=$ preparation diameter, $\mathrm{H}=$ occlusocervical dimension and $\mathrm{L}=$ width of the finish line. The three variable dimensions are the TOC (total occlusal convergence), the FL (finish line) and COF (curvature of the occlusal face).

For each preparation design, five specimens were milled from aluminum rods (Al 6060) with a 5-axes DMU40 milling machine (Deckel Maho Gildemeister, Bielefeld Germany) with $2 \mu \mathrm{m}$ accuracy (manufacturer data). The cylindrical basis of the rod was maintained for use as a sample holder

\subsubsection{Ceramic caps}

The ceramic caps were made with the Cerec System (Sirona Dental System, Bensheim, Germany). One optical imprint was recorded for each preparation [P1-P4] with the "blue cam" Cerec camera. The same external geometry was designed for all of the preparations using 3.80 Cerec CAD/ CAM software. The minimum thickness facing the occlusal area was set at $2 \mathrm{~mm}$. The software was programmed to provide a dento-prosthetic spacing of $100 \mu \mathrm{m}$ and a peripheral joint of $40 \mu \mathrm{m}$. The caps were manufactured using a Cerec MC-XL milling unit with Vita MarkII ceramic blocks (Vita Zahnfabrik, Bad Sackingen, Germany) and were not glazed nor polished. The caps were etched on their internal 
Fig. 1 Fabrication of a sample: a P2 Design, b P2 milled in aluminum rod, $\mathbf{c}$ Optical print of $\mathrm{P} 2$, $\mathbf{d}$ ceramic caps glued on aluminum specimen

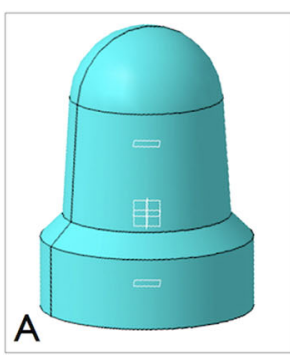

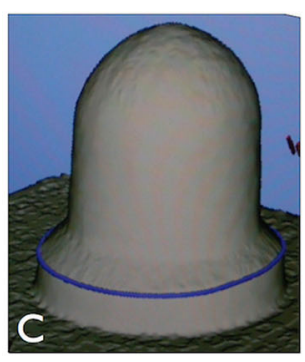

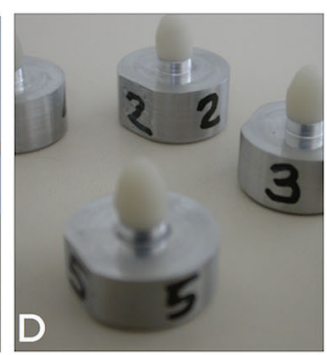

surface using a $5 \%$ hydrofluoric acid gel for $1 \mathrm{~min}$ (Vita Etch, Vita Zahnfabrik, Bad Säckingen, Germany) and bonded onto the preparations with Relyx-Unicem Applicap (3 M ESPE Dental Division, St. Paul, Minn). The caps were subsequently cured using a Swiss Master Light curing unit (E.M.S., Nyons, Switzerland) according to the manufacturer's recommendations ( $4 \mathrm{~s}$ at $3000 \mathrm{~mW} / \mathrm{cm}^{2}$ per face). Figure 1 shows the different stages of the P2 sample fabrication.

\subsubsection{Mechanical testing and measurement systems}

The samples were subjected to compression until fracture. Compression tests were performed with the Dartec mechanical testing system (TestRessources Inc., Shakopee, Minn.) driven by Tematest software (Tema Concept, Chanteloup-les-Vignes, France) with regulated loading speeds. A TC4 load cell (500 daN) (Nordic Transducer, Hadsund, Denmark) coupled with an LVDT (linear variable differential transformer) displacement sensor with a $1 \mathrm{~mm}$ range was used (L10R transducer, RDP Electrosense, Pottstown, Pa). A $10 \mathrm{~N}$ preload was applied to the sample prior to the test. Progressive loading was performed, while the LVDT sensor monitored the compressive displacement response of the sample. During the test, the force and displacement were continuously recorded, and the rupture force values were documented.

\subsection{In silico study}

The Finite Element simulation of the compression tests was performed using commercial FE software (ANSYS v14, ANSYS Inc., Canonsburg, PA, USA). A FE model was produced for each of the four studied designs. The models take into account three components: the aluminum preparation, the ceramic cap and the glue. The shapes and dimensions of these components were set in the FE models based on the geometrical measurements of the samples. The axial symmetry of both the sample geometry and the load enabled a 2D axisymmetric FEA. Accordingly, the components of the model were meshed using quadrilateral $2 \mathrm{D}-8$ node elements (PLANE183) with axisymmetric behavior. This modeling produced a total of 3128 elements and 9336 nodes. The elements have two degrees of freedom (translations) at each node and are based on quadratic displacement functions that are well suited for curved geometries. The boundary conditions of the model correspond to the mechanical compression test conducted. The basis of the preparation was clamped and the top face of the ceramic cap was submitted to a uniformly distributed axial compression force.

In this FEA, all the materials were assumed to be homogeneous, isotropic, and linearly elastic during deformation until fracture of the ceramic cap. The contact between the glue and aluminum preparation was modeled as sliding (frictionless), and the contact between the glue and ceramic was assumed perfectly bonded (continuity of the displacements at the coincident nodes). The Young's modulus of the materials was set at $70 \mathrm{GPa}$ for aluminum, 63 $\mathrm{GPa}$ for ceramic and $8.4 \mathrm{GPa}$ for glue. The Poisson's ratio was set at 0.3 for all the materials. For the ceramic, 290 $\mathrm{MPa}$ was used for the compressive strength and $113 \mathrm{MPa}$ was used for the tensile strength in the FEA.

The FE simulation of the compression test required nonlinear solving because of the presence of contact in the model; thus, automatic time stepping was used. At each computational step, the stress distribution was evaluated in all of the materials and all directions (radial, axial and hoop) (Fig. 2). The compression force was incrementally increased until one of the stress components inside the ceramic locally reached the compressive or tensile strength of the material. Next, the stress distributions were compared between the four models, and the computed maximum forces were compared with the measured rupture forces.

\subsection{Clinical study}

\subsubsection{Patients and controls}

From 2003 to 2008, 497 patients received 580 ceramic restorations. Patients were followed for 6 years, and the last patient follow up occurred in 2014. Exclusion criteria for the study consisted of tooth absence on the opposing arch, wisdom tooth, parafunctions, bruxism, psychological disorders, and an inability to return for follow up visits for 5 years after prosthesis placement. Molars restored by 
Fig. 2 FE model for the ceramic restoration with the $\mathrm{P} 1$ design. The model takes into account three components: the preparation, the ceramic cap and the glue. a Sagittal cut. Red arrows indicate the orientation of the compressive force. $\mathbf{b}$ Expanded volume (3/4 expansion). Black arrows indicate the direction of the exerted stresses

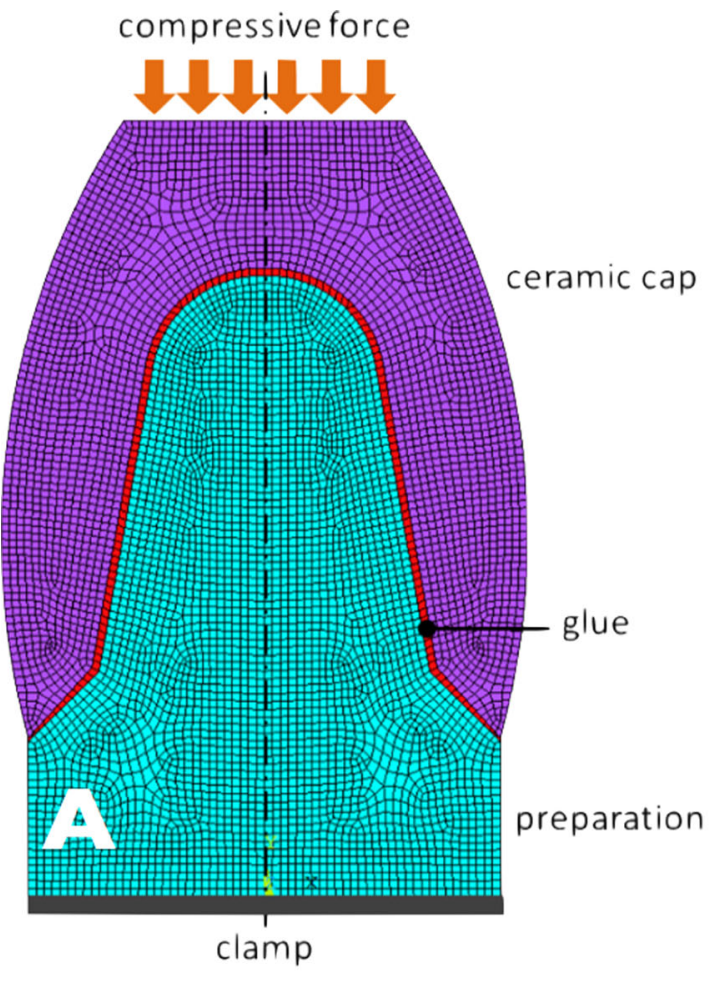

endocrowns are not included in this study. During the subsequent 5 years that followed each crown procedure, each patient was examined at least once a year in conjunction with other treatments or routine visits. The latest crowns included in this study were inserted in 2008. The last patient follow up occurred in 2015 .

Failure criteria included a loss of the restoration, partial or total tooth and/or ceramic fracture, development of marginal caries, and marginal endodontic complications.

\subsubsection{Preparation of teeth}

Teeth were prepared in accordance with the best results obtained by in vitro and in silico studies. So the P4 shape served as the model (Fig. 3).

All teeth were prepared with green ring diamond burs (NTi-Kahla GmbH Rotary Dental Instruments, Kahla, Germany). The cervical margins were polished with red ring diamond burs (NTi-Kahla GmbH Rotary Dental Instruments, Kahla, Germany). The cervical limit was 800 $\mu \mathrm{m}$ minimum right shoulder widths. The total occlusal convergence of the axial walls was $7^{\circ}$ with a minimum height of $4 \mathrm{~mm}$. A minimum of $1.5 \mathrm{~mm}$ occlusal reduction oriented parallel to the occlusal plane was used. The occlusal surfaces were flat. If necessary, composite was used to replace the loss of substance on the coronal segment. In cases of severe damage, fiber posts (Apoll, Champagnole France) were luted into the canals using Rely
X UniCem (3 M Espe Dental Division, St. Paul, MN, USA). The composite was used to build the coronal section.

\subsubsection{CAD/CAM procedure}

The CAD/CAM procedure was performed using the CEREC system (Sirona Dental Systems GmbH Benshein Germany). An optical impression was made after tooth preparation using the "red cam" camera.

The software was programmed to obtain a $100-\mu \mathrm{m}$ dental-prosthetic thickness with a cervical junction $40 \mu \mathrm{m}$ thick and $800 \mu \mathrm{m}$ wide. The minimum ceramic thickness was $1.5 \mathrm{~mm}$ for the occlusal surface. The milling unit was CEREC MC.

\subsubsection{Ceramics}

Vita Mark II (Vita Zahnfabrik, Bad Sackingen, Germany) ceramic blocks were used. All restorations were glazed using an Akzent glaze and Atmomat furnace (Vita Zahnfabrik, Bad Sackingen, Germany) according to the manufacturer's instructions. Prosthetic intrados were etched with Vita Etch hydrofluoric acid (Vita Zahnfabrik, Bad Sackingen, Germany) according the manufacturer's instructions.

\subsubsection{Bonding}

Enamel was etched on prepared teeth with orthophosphoric acid (ScothBond Gel, 3 M Espe Dental Division, St. Paul, 
Fig. 3 Teeth preparations in accordance with the $\mathrm{P} 4$ principle. a a second upper molar, $\mathbf{b}$ a second upper premolar

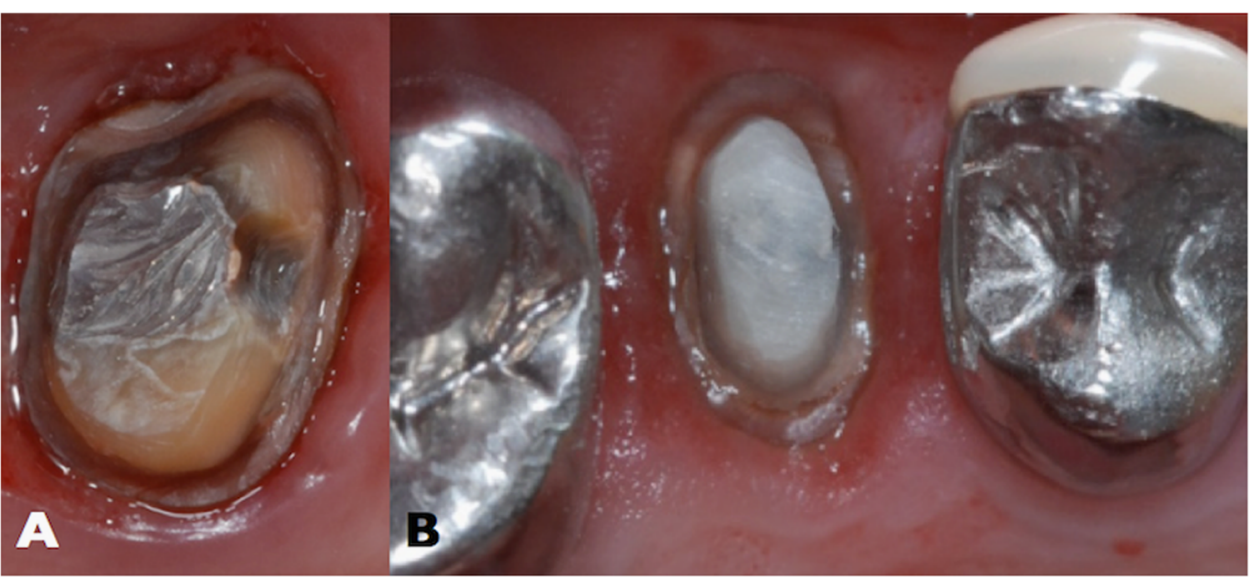

MN, USA). After preparation of the ceramic according to the manufacturer's suggestions and finishing (checking occlusion and contact surfaces), bonding was completed using self-adhesive cement (Relyx Unicem, $3 \mathrm{M}$ Espe Dental Division, St. Paul, MN, US. with the light Curing units (LCUs), Aurys (Degré K, Paris France) and Swiss Master Light (EMS, Nyon, Switzerland).

\section{Results}

\subsection{In vitro study}

The results for the in vitro and in silico studies are shown on Table 2.

The mean rupture values are $1.048 \mathrm{kN}$ for $\mathrm{P} 1$ and 1.844 $\mathrm{kN}$ for $\mathrm{P} 4$, which equates to a difference of approximately $80 \%$.

For all tests, the fracture occurred as a complete rupture that caused total destruction of the ceramic cap (Fig. 4). The ceramic shattered into a multitude of fragments of variable sizes.

It was noted that there is trace of glue on the intrados of the fragments of the ceramic cap but not on the surface of the preparation.

\subsection{In silico study}

The stress distributions at fracture in the ceramic caps for the FE rupture forces are reported in Fig. 5. The P4 design led to the best resistance.

As shown in Fig. 2, the P1 restoration presented the lowest rupture force value $(1.223 \mathrm{kN})$, and the $\mathrm{P} 4$ restoration exhibited the highest rupture force value $(2.253 \mathrm{kN})$. The difference in these rupture force values is approximately $80 \%$.

The four designs exhibited different fracture locations in the ceramic cap and rupture modes. For P1, rupture stress
Table 2 Results of the experimental tests (in vitro) and the FE study with respect to preparation geometries Rupture force of P1, P2, P3, P4 $(\mathrm{kN})$, the SD (standard deviation) and the F.E rupture forces $(\mathrm{kN})$

\begin{tabular}{lllll}
\hline Sample & P1 & P2 & P3 & P4 \\
\hline Rupture force $(\mathrm{kN})$ & 1.048 & 1.255 & 1.744 & 1.884 \\
SDN (rupture force) $(\mathrm{kN})$ & 0.057 & 0.099 & 0.256 & 0.344 \\
FE force rupture $(\mathrm{kN})$ & 1.223 & 1.457 & 1.644 & 2.263 \\
\hline
\end{tabular}

was reached in tension at the cervical limit. For P2, rupture stress was reached in tension at the intrados of the occlusal face and at the cervical limit. For P3, the rupture stress was reached in tension at the intrados of the occlusal face. For $\mathrm{P} 4$, the rupture stress was reached simultaneously in tension at the intrados of the occlusal face and in compression at the extrados of the occlusal face. Within the limitation of the FEA, preparation designs with a $45^{\circ} \mathrm{FL}$ (shoulder angulation) led to tensile rupture of the ceramic cap located at the cervical limit and the intrados of the occlusal face. Preparations with a $90^{\circ} \mathrm{FL}$ (chamfer angulation) showed improved strength. A lower TOC also improved strength.

\subsection{In vivo study}

All cases of ceramic fractures induced only a partial destruction of the restoration (Fig. 6).

A total destruction of restoration, including tooth fractures or endodontic complications, was not observed. Out of the 580 restorations, only 6 failures occurred resulting (Table 3) in a success rate of $98.97 \%$ (Table 4).

Three of them appeared during the 1st month, and one at the 6th month follow-up. One failure appeared 1 year after the restoration, and the last failure occurred 2 years after the restoration. After 2 years, no additional failures were observed.

It was noted two failures on 366 premolars $\left(\mathrm{N}^{\circ} 24\right.$ and $35)$ respectively at the 1 st month and the 12 th month. This 


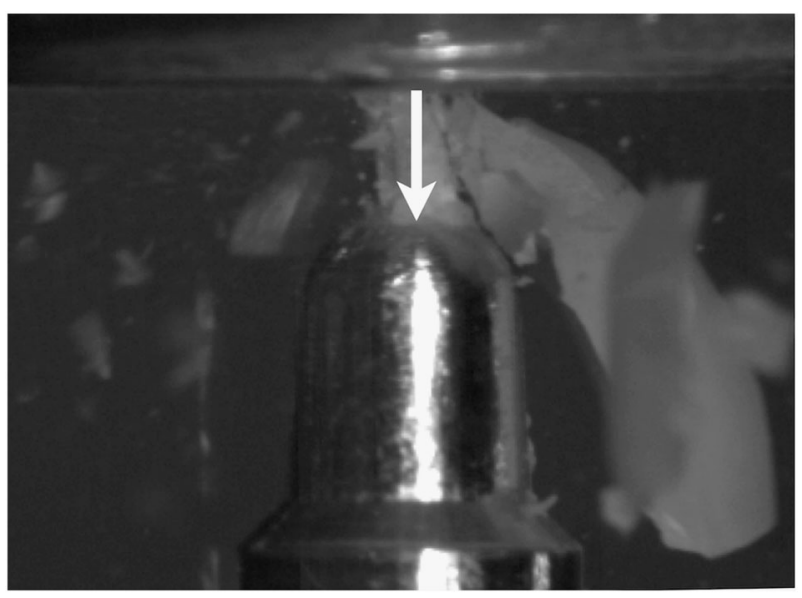

Fig. 4 Ruptures of P2 restorations. White arrows indicate the force axis. The ceramic shattered in a multitude of fragments causing the total destruction of the cap

Fig. 5 For each direction (radial, axial, hoop), compressive stresses

(respectively tensile) are marked in blue (respectively in red) and correspond to negative (respectively positive) values

P1 gives a percentage of success of $99.46 \%$. It was noted four failures on 214 molars $(16,17,27,47)$ that give a percentage of success of $98.12 \%$ (Figs. 7, 8).

Three failures occurred on the second molars $(17,27,47)$, and only one on a first molar (16). Three failures occurred on the upper maxillary and only one on the mandible.

\section{Discussion}

In all the three studies, in silico, in vitro and in vivo, results look excellent.

If the stress distributions at ceramic fracture are compared between the four models, $\mathrm{P} 4$ design exhibits a better strength. Effect of TOC, FL and COF were discussed in literature but seldom for their influence on the mechanical
P2

$(1.293 \mathrm{kN})$

(1.293

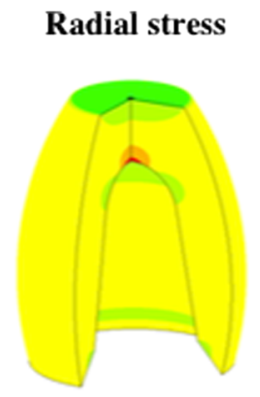

Axial stress

\section{Hoop stress}
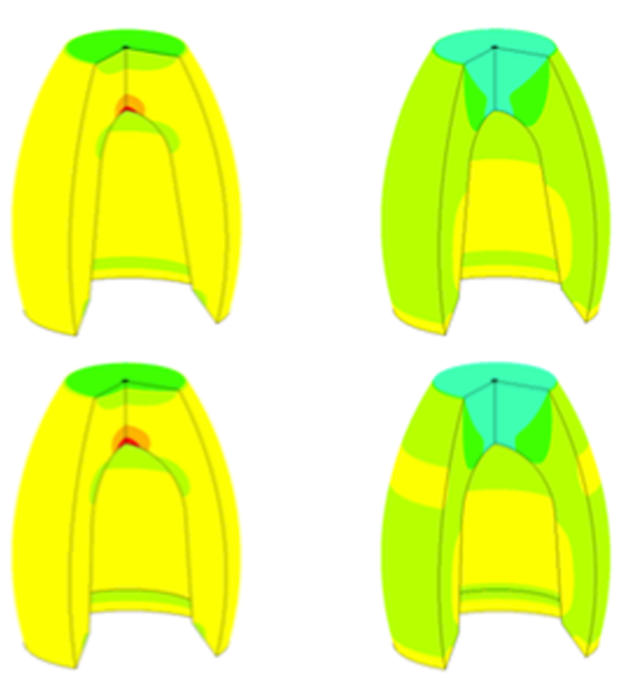

P3

$(1.644 \mathrm{kN})$

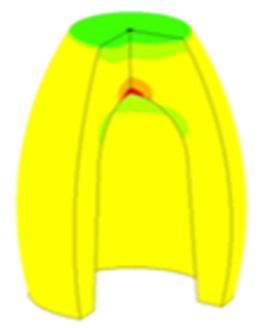

P4

$(2.163 \mathrm{kN})$

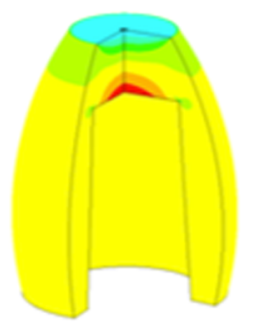

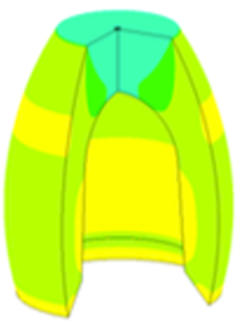
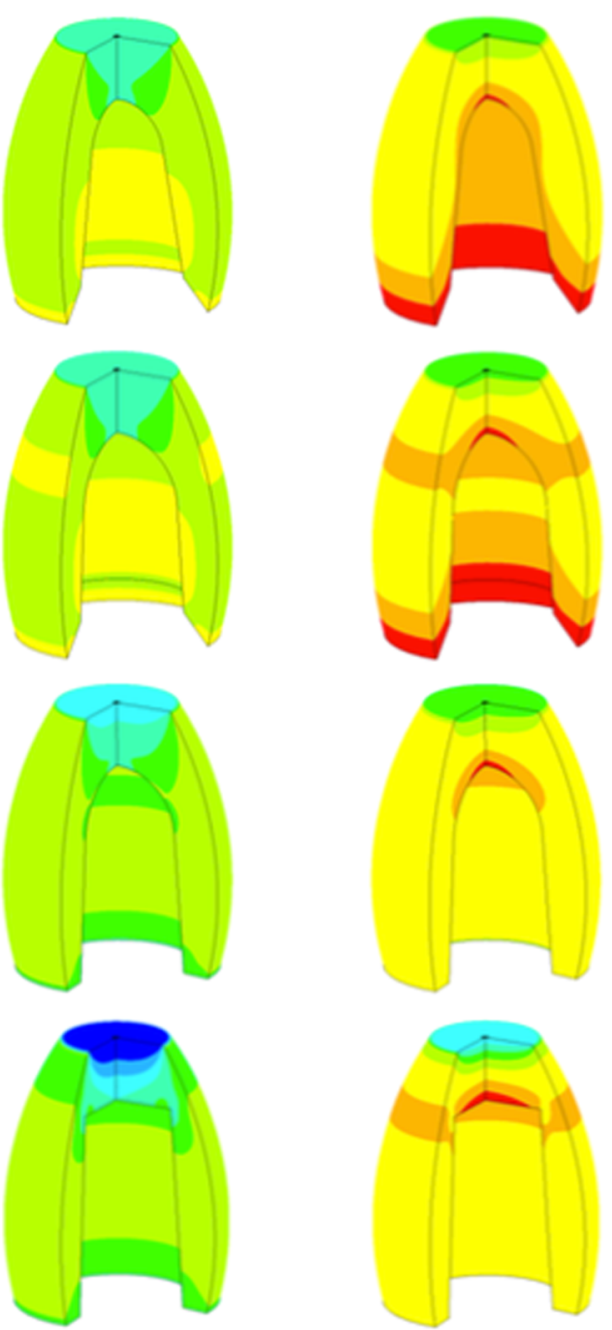
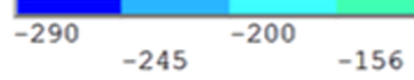

$-111$ $-66$ 
Fig. 6 Failures: a upper second premolar, b first upper molar

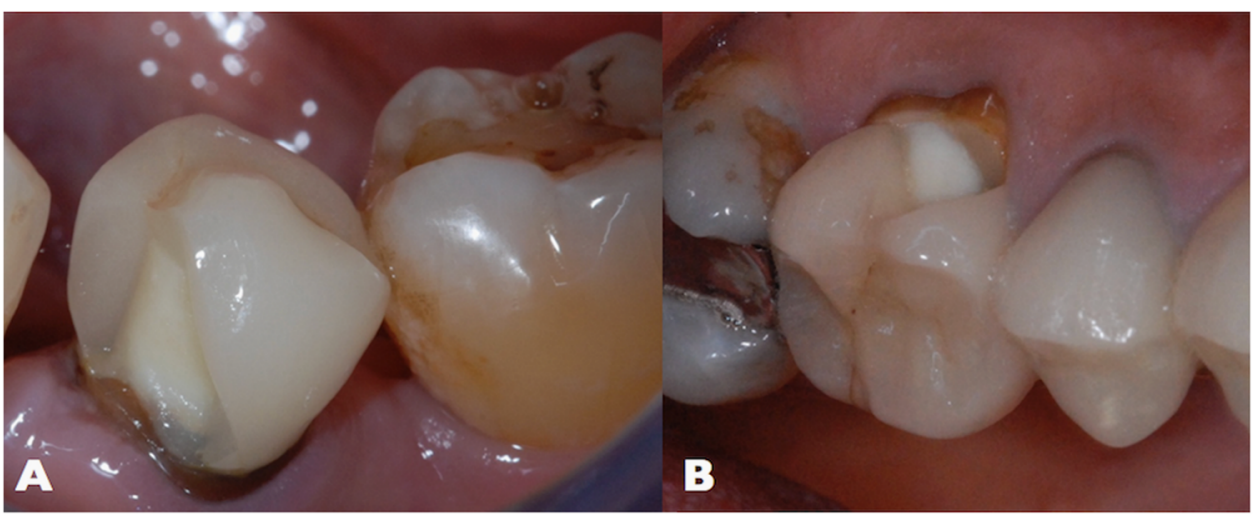

Table 3 Successes and failures for premolars and molars, failures apparition

\begin{tabular}{lllll}
\hline Tooth $\mathrm{N}^{\circ}$ & Success & Failure & Total & Failure apparition (month) \\
\hline 14 & 49 & - & 49 & - \\
15 & 31 & - & 31 & - \\
24 & 75 & 1 & 76 & 1 \\
25 & 54 & - & 54 & - \\
34 & 37 & - & 37 & - \\
35 & 55 & 1 & 56 & 12 \\
44 & 24 & - & 24 & - \\
45 & 39 & - & 39 & - \\
16 & 40 & 1 & 41 & 6 \\
17 & 13 & 1 & 14 & 1 \\
26 & 28 & - & 28 & - \\
27 & 8 & 1 & 9 & 24 \\
36 & 49 & - & 49 & - \\
37 & 18 & - & 18 & - \\
46 & 38 & - & 38 & - \\
47 & 16 & 1 & 17 & 1 \\
\hline
\end{tabular}

strength of the materials [19]. The influence of the TOC on the mechanical principle of "retention-stabilization" $[20,21]$ is well known; however, less is known about its impact on the mechanical response of glass ceramic under load. Prothero in 1923 [22] and Jorgensen in 1955 recommended $2-5^{\circ}$ for the TOC. Recently, Wilson and Chan [23] reported that maximal tensile retention occurred between 6 and $12^{\circ}$. Annerstedt et al [24] reported that the mean TOC achieved by dentists in clinical practice was approximately $20^{\circ}$. For that the TOC values of $7^{\circ}$ and $21^{\circ}$ were chosen for this study. Occlusal face geometry, the COF, is an important parameter that affects the degree of stress concentration [25]. Thus, rounded occlusal faces with flat occlusal faces were compared. The effect of the FL size on the behavior of loaded ceramic was previously reported [26]; however, the possible role of its angulations was not clearly analyzed.
Table 4 Global results in percentages

\begin{tabular}{llll}
\hline Teeth & Restorations & Failures $(\%)$ & Success $(\%)$ \\
\hline Premolars & 366 & 0.54 & 99.46 \\
Molars & 214 & 1.88 & 98.12 \\
Total & 580 & 1.03 & 98.97 \\
\hline
\end{tabular}

Based on the literature [27, 28], we studied two different FL values: $45^{\circ}$ and $90^{\circ}$

The four designs exhibited different fracture locations in the ceramic cap and rupture modes [29]. For P1, rupture stress was reached in tension at the cervical limit. For P2, rupture stress was reached in tension at the intrados of the occlusal face and at the cervical limit. For P3, the rupture stress was reached in tension at the intrados of the occlusal face. For P4, the rupture stress was reached simultaneously in tension at the intrados of the occlusal face and in compression at the extrados of the occlusal face. Within the limitation of the FEA, preparation designs with a $45^{\circ} \mathrm{FL}$ (shoulder angulation) led to tensile rupture of the ceramic cap located at the cervical limit and the intrados of the occlusal face. Preparations with a $90^{\circ}$ FL (chamfer angulation) showed improved strength. A lower TOC also improved strength. From a mechanical point of view, the influence of the FL and TOC can be explained by the fact that a high TOC value and a sloped FL $\left(45^{\circ}\right)$ induce an opening of the ceramic cap under axial loading. This type of deformation leads to tensile stresses at the cervical limit and the intrados of the occlusal face that can break the ceramic. In contrast, a $90^{\circ}$ cervical shoulder can favor compression by acting as a "lock" at the opening of the ceramic that increases its strength. The flat occlusal face of P4 showed increased strength due to a reduction in the stress in the intrados of the occlusal face of the ceramic cap.

For the in vitro study, the result for each preparation was significantly different. The comparison of the mean value of the rupture forces showed that $\mathrm{P} 1$ had the lowest value $(1.048 \mathrm{kN})$ and $\mathrm{P} 4$ exhibited the highest value $(1.884 \mathrm{kN})$. 
Fig. 7 Success on an upper premolar: a clinical case, b tooth preparation, $\mathbf{b}$ restoration in place
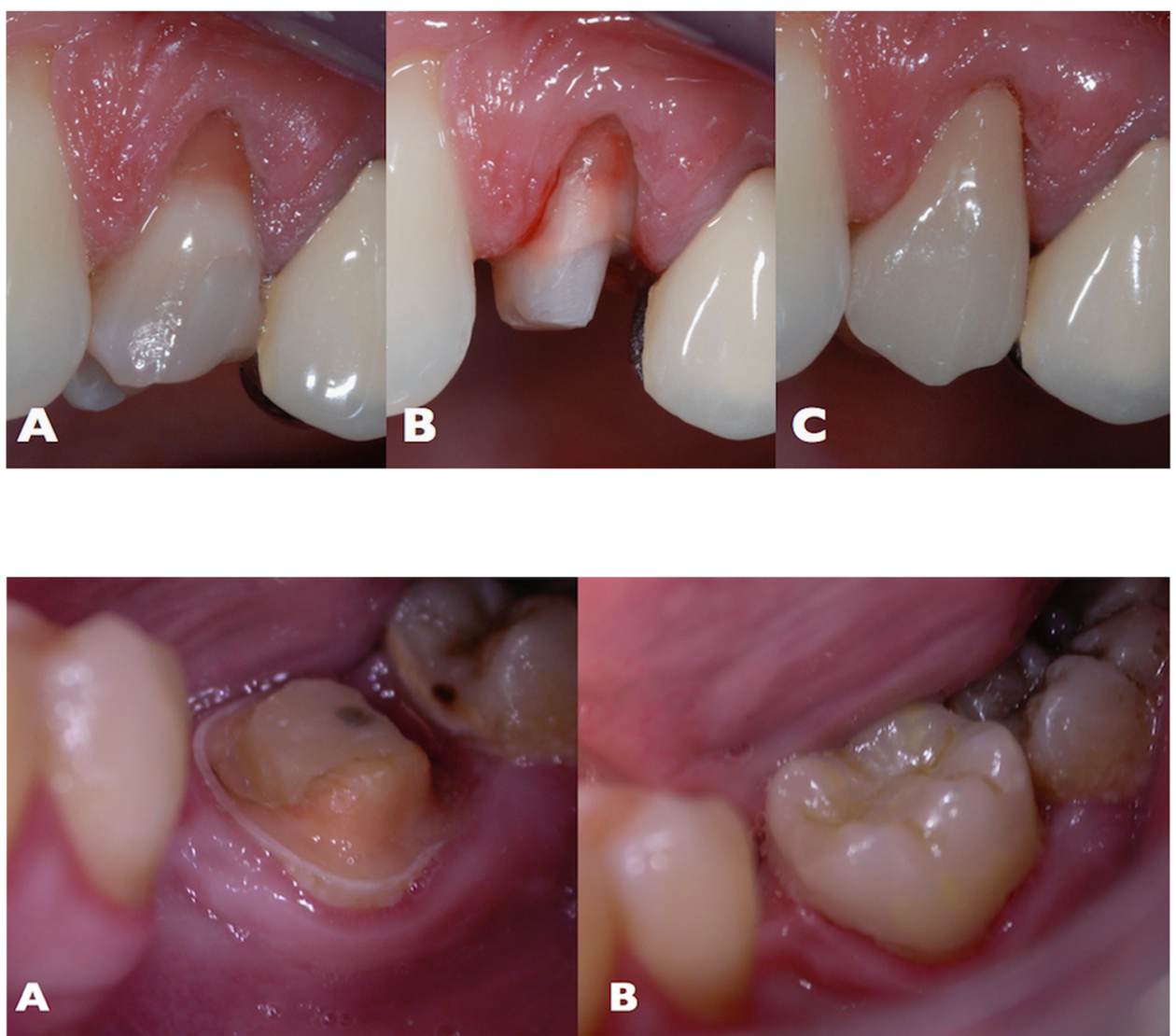

Fig. 8 Success on an upper molar: a preparation, $\mathbf{b}$ restoration in place
Small standard deviations highlight the influence of geometry on restoration resistance. The simple variation of TOC, FL, and COF provided an approximate $80 \%$ increase in resistance. This considerable increase is in accordance with the FEA.

Notably, this improvement in mechanical properties can be obtained by a simple change in clinical practice that avoids the excessive mutilation required to adapt to the thickness of the ceramic ${ }^{36}$, the use of harder materials that are not compatible with the stiffness of the opposing teeth creating an imbalance in wear, and differences in the stress to the supporting tooth which must accommodate and not suffer or transmit stresses.

The in vitro study confirmed the FEA. The measured rupture forces are in agreement with the computed values from the FEA (Fig. 9). Specifically, the rank of the four preparations and their respective strength was corroborated by the FEA computations.

The $\mathrm{P} 4$ preparation design was determined to be the "definitive concept" of tooth preparation that was applied to all the preparations of the clinical study.

A total destruction of restoration, including tooth fractures or endodontic complications, was not observed. This result indicates that the underlying tooth structure was not damaged, which is essential for the conservation of teeth on the arch. The fracture of a reconstruction is bothersome, but tooth damage is far worse. Underlying damage can proliferate with no symptoms and often create considerable deterioration. It is dangerous to believe that a tooth is out of danger as long as it is covered by a "silent" restoration. A basic question can be asked: "Must my restoration or the support of my restoration endure forever?" In the cases of partial fracture of the restoration, removal of a feldspar ceramic is easy because of its hardness being close to that of enamel. The action of the clinician is then easier and prevents damage to the underlying tooth. This is not the case with harder materials, such as zirconia [30] or metal and even more if it is necessary to remove a post sealed or glued in the root of the tooth.

The majority of failures (33\%) occurred during the 1st month. Four out of six failures occurred during the 1st year, and no additional failures were observed after 2 years. The "immediate" failures can be considered as an advantage. Those failures were likely due to a problem with ceramic thickness, occlusion constraints, preparation design, or an error in the luting protocol [31]. This type of mechanical problem can be immediately analyzed and corrected without damage to the underlying tooth.

It is interesting to compare the results of this study with those obtained for molar restoration using lithium disilicate 


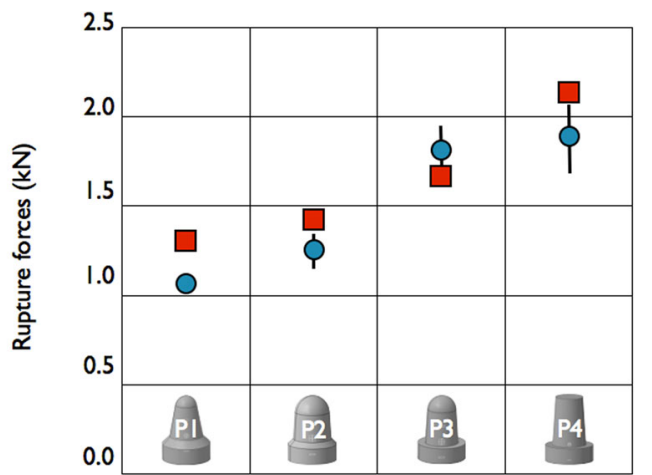

Rupture force | Standart deviation $\quad \square$ Finite element rupture force

Fig. 9 Rupture forces with SD and computed values from the FEA

because it is much harder than feldspar ceramic [32]. Lithium disilicate all-ceramic restorations exhibited satisfactory clinical performance with an estimated survival probability of $87.1 \%$ over 104.6 months [33, 34]. However, out of 214 feldspathic-reconstituted molars, only 4 failures occurred, resulting in a survival rate of $98.12 \%$ over 72 months

If we consider the teeth using categories, the first lower molar was the most reconstituted with 87 restorations and no failures. Out of the 366 premolars reconstituted, there were only two failures $(0.54 \%$ failure rate). This rate is much lower than observed for IPS-Empress conventional partial ceramic restorations (inlays/onlays) [35] at 4.5 years $(4 \%)$ and at 7 years $(9 \%)$.

The low rate of failure obtained during testing was expected after the in silico and in vitro studies. The adhesion capacity of the adhesive joint plays an important role. It is likely that the rupture of the adhesive joint determines the debonding between the ceramic and its support, thereby causing the fracture. This hypothesis is supported by the fact that after fracture there is no glue on the support.

From a mechanical point of view, the influence of the TOC and FL could be explained by the fact that high TOC values and sloped $\mathrm{FL}\left(45^{\circ}\right)$ might induce an opening of the ceramic under axial loading. That might cause tensile stresses inside its internal area, which are known to favor the breaking of the ceramic. Besides, low values of the COF could result in stress concentration under the ceramic cap due to its punching by the infrastructure.

The FEA confirms that $\mathrm{P} 4$ design mainly accommodates compressive stresses, which is not the case with the other preparations designs (Fig. 5).

Thus, tooth preparation should not be considered as a reduction of leaving space for "reconstruction materials," but as an "optimal reduction" that allows for the best strength, accommodation for reconstruction and materials chosen.

\section{Conclusion}

Within the limitation of this study it is possible to conclude that the variations of geometric design parameters have a significant influence on the strength of the ceramic. A good combination of these parameters allows for reconstructions with a mechanical breaking up to $80 \%$ higher than that of a non-appropriate combination. These results are confirmed in clinical practice. This study could be complemented by the analysis of additional shapes and the specific influence of the glue itself. This study confirms that dental preparations should no longer be considered as simple geometric shapes defined mainly by the "retention-stabilization" principle. They must be understood as architectural constructions favorably distributing load transfers, in line with new ceramic materials for reconstruction, and fixing according to bio-integration concept.

\section{Compliance with ethical standards}

Conflict of interest The authors declare that they have no competing interests.

\section{References}

1. Simonsen RJ. Conservation of tooth structure in restorative dentistry. Quintessence Int.1985;16:15-24.

2. Simonsen RJ. The preventive resin restoration: a minimally invasive, non metallic restoration. Compendium. 1987;8:428-32.

3. Goodacre CJ, Campagni WV, Aquilino SA. Tooth preparations for complete crowns: an art form based on scientific principles. J Prosthet Dent. 2001;85:363-76.

4. Dawson AS, Cardaci SC. Endodontics versus implantology: to extirpate or integrate? Aust Endod J. 2006;32:57-63.

5. McCrea SJ. Advanced peri-implantitis cases with radical surgical treatment. J Periodontal Implant Sci. 2014;44:39-47.

6. Mante FK, Ozer F, Walter R, Atlas AM, Saleh N, Dietschi D, et al. The current state of adhesive dentistry: a guide for clinical practice. Compend Contin Educ Dent. 2013;34:2-8.

7. Magne P, Douglas WH. Rationalization of esthetic restorative dentistry based on biomimetics. J Esthet Dent. 1999;11:5-15.

8. Bazos P, Magne P. Bio-Emulation: biomimetically emulating nature utilizing a histoanatomic approach; visual synthesis. Int $\mathbf{J}$ Esthet Dent. 2014;9:330-52.

9. Harkness JM. An idea man. Otto Herbert Schmitt. IEEE Eng Med Biol Mag. 2004;23(6):20-41.

10. Julien V, Bogatyreva O, Bogatyrev N, Bowyer A, Pahl A-K. Biomimetics: its practice and theory. $\mathrm{J}$ R Soc Interface. 2006;3:471-82.

11. Bazos P, Magne P. Bio-Emulation: biomimetically emulating nature utilizing a histoanatomic approach; visual syntesis. Int $\mathbf{J}$ Esthet Dent. 2014;9(3):330-52.

12. Barak MM, Geiger S, Chattah NLT, Shahar R, Weiner S. Enamel dictates whole tooth deformation: a finite element model study validated by a metrology method. J Struct Biol. 2009;168:511-20.

13. Zaslanski P, Friesem AA, Weiner S. Structure and mechanical properties of the softzone separating bulk dentin and enamel in 
crowns of human teeth: insight into tooth function. J Struct Biol. 2006;153:188-99.

14. Baroudi K, Ibraheem SN. Assessment of chair-side computeraided design and computer-aided manufacturing restorations: A review of the literature. J Int Oral Health. 2015;7:96-104.

15. Burke FJ, Qualtrough AJ, Hale RW. The dentin-bonded ceramic crown: an ideal restoration? Br Dent J. 1995;179:58-63.

16. Fages M, Slangen P, Raynal J, Corn S, Turzo K, Margerit J, et al. Comparative mechanical behavior of dentin enamel and dentin ceramic junctions assessed by speckle interferometry (SI). Dent Mater.. 2012;28:229-38.

17. El-Mowafy O, Brochu JF. Longevity and clinical performance of IPS- Empress ceramic restorations-a literature review. J Can Dent Assoc. 2002;68:233-7.

18. Zahran M, El-Mowafy O, Tam L, Watson PA, Finer Y. Fracture strength and fatigue resistance of all-ceramic molar crowns manufactured with CAD/ CAM technolog. J Prosthodont. 2008;17:370-7.

19. Friedlander LD, Munoz CA, Goodacre CJ, Doyle MG, Moore BK. The effect of tooth preparation design on the breaking strength of Dicor crowns: Part 1. Int J Prosthodont. 1990;3:159-68.

20. Schilinburg HT, Hobo S, Whitsett LD, Jacobi R, Brackett SE. Fundamentals of fixed prosthodontics. Chicago: Quintessence; 1997. p. 11-72.

21. Prothero JH. Prosthetic dentistry. Chicago, IL: Medico-Dental Publishing Co; 1923. p. 742. 1099, 1101-6, 1128-38

22. Jorgensen KD. The relationship between retention and convergence angle in cemented veneer crowns. Acta Odontol Scand. 1955;13:35-40.

23. Wilson AH Jr, Chan DC. The relationship between preparation convergence and retention of extracoronal retainers. J Prosthodont. 1994;3:74-8.

24. Annerstedt A, Engström U, Hansson A, Jansson T, Karlsson S, Lilijhagen $\mathrm{H}$, et al. Axial wall convergence of full veneer crown preparations. Documented for dental students and general practitioners. Acta Odontol Scand. 1996;54:109-12.
25. Sornsuwan $T$, Swain MV. Influence of occlusal geometry on ceramic crown fracture; role of cusp angle and fissure radius. J Mech Behav Biomed Mater. 2011;4:1057-66.

26. Syu JZ, Byrne G, Laub LW, Land MF. Influence of finish-line geometry on the fit of crowns. Int J Prosthodont. 1993;6:25-30.

27. Jalalian E, Aletaha NS. The effect of two marginal designs (chamfer and shoulder) on the fracture resistance of all ceramic restorations, Inceram: an in vitro study. J Prosthodont Res. 2011;55:121-5.

28. Addison O, Sodhi A, Fleming GJ. Seating load parameters impact on dental ceramic reinforcement conferred by cementation with resin-cements. Dent Mater. 2010;26:915-21.

29. Thompson VP, Rekow DE. Dental ceramics and the molar crown testing ground. J Appl Oral Sci. 2004;12:26-36.

30. Dejak B, Młotkowski A. 3D-Finite element analysis of molars restored with endocrowns and posts during masticatory simulation. Dent Mater. 2013;29:309-17.

31. de Almeida AA Jr, Munoz Chavez OF, Galvao BR, Adabo GL. Clinical fractures of veneered zirconia single crowns. Gen Dent. 2013;61:17-21.

32. Zahran M, El-Mowafy O, Tam L, Watson PA, Finer Y. Fracture strength and fatigue resistance of all-ceramic molar crowns manufactured with CAD/CAM technology. J Prosthodont. 2008;17:370-7.

33. Fischer $\mathrm{H}$, et al. Chemical strengthening of a dental lithium disilicate glass-ceramic material. J Biomed Mater Res A. 2008;87:582-7.

34. Toman M, Toksavul S. Clinical evaluation of 121 lithium disilicate all-ceramic crowns up to 9 years. Quintessence Int. 2015;46:189-97.

35. Beier US, Kapferer I, Burtscher D, Giesinger JM, Dumfahrt H, Inlay onlay survival rates. Clinical performance of all-ceramic inlay and onlay restorations in posterior teeth.Int J Prosthodont. 2012;25:395-2 